\title{
ON POINT DISSIPATIVE N-DIMENSIONAL SYSTEMS OF DIFFERENTIAL EQUATIONS WITH QUADRATIC NONLINEARITY
}

\author{
ANIL K. BOSE \\ ALAN S. COVER \\ JAMES A. RENEKE
Department of Mathematical Sciences
Clemson University
Clemson, SC 29634-1907

(Received October 17, 1991)

ABSTRACT. Known sufficient conditions for quadratic dynamical system $x^{\prime}=A x+f(x)$ to be point dissipative given in terms of $A$ and $f$ for dimensions 2 and 3 are extended to allow for more general forms for the nonlinear term $f(x)$. Furthermore, the conditions extend to $n$ dimensions when $f$ is quadratic with zero set an (n - 1)-dimensional hyperplane.

\section{INTRODUCTION}

We are concerned with a class of vector equations of the form $x^{\prime}=A x+f(x)$ where the nonlinear term $\mathbf{f}(\mathbf{x})$ is quadratic of the form

$$
f(x)=\left[\begin{array}{c}
x^{T} C_{1} x \\
\cdot \\
\cdot \\
\cdot \\
x^{T} C_{n} x
\end{array}\right]
$$

The $n \times n$ matrices $\left\{C_{i}\right\}$ are assumed symmetric with the orthogonality property $x^{T} f(x)=0$ for all $x$. If $x^{T} f(x)=0$ for all $x$ we say that $f$ is a conservative function. Note that if $x^{\prime}=f(x)$ with $f$ conservative then $\|x\|^{2}$ is constant. The problem is to determine conditions on $A$ and $f$ sufficient to have the system point dissipative, i.e., which guarantee the existence of a bounded region $R$ with the property that every trajectory of the system eventually enters and remains within $R$ [2].

Consider the Lyapunov function $V(x)=\frac{1}{2}(x-\alpha)^{T}(x-\alpha)$ for the system. For large $\|x\|$ the quadratic terms $x^{T} A x-\alpha^{T} f(x)$ dominate $\frac{d(V(x(t)))}{d t}$. Therefore our quest is to find conditions on 
A and $\mathbf{f}$ for which there is an admissible $\alpha$ which makes these terms a negative definite function. If there exists an admissible $\alpha$ then a classic result [ 4 ] implies the system is point dissipative.

A necessary condition for the existence of an admissible $\alpha$ is that $x^{\mathrm{T}} \mathrm{Ax}<0$ for each nontrivial $x$ in the zeros of $f$. We have shown for $n=2$ and 3 [ 1 ] that this condition is also sufficient. Settling the obvious conjecture for all $\mathrm{n}$ bogs down in a proliferation of cases. However, we have shown in this paper that the necessary condition is sufficient for the simplest $\mathrm{n}$-dimensional case, namely, when the zeros of $f$ form an ( $n$ - 1)-dimensional hyperplane.

Finally, we extend the notion of an admissible $\alpha$ to provide sufficient conditions for systems $\mathbf{x}^{\prime}=A \mathbf{x}+\mathbf{f}(\mathbf{x})+\mathbf{g}(\mathbf{x})$ to be point dissipative when $\mathbf{f}$ is quadratic but not conservative and $\mathbf{g}$ is not quadratic. If there is a positive definite matrix $S$ such that $S f$ is conservative then a vector $\alpha$ is admissible for the dynamical system $x^{\prime}=A x+f(x)$ if $x^{\mathrm{T}} \mathbf{S A x}-\alpha^{\mathrm{T}} \mathrm{Hf}(\mathbf{x})$ is a negative definite function where $H^{\mathrm{T}} H=S$. If $\alpha$ is an admissible vector for the system $x^{\prime}=A x+f(x)$ then the system $x^{\prime}=A x+f(x)+g(x)$ is point dissipative if there is an ordered triple $(\varepsilon, C, M)$ such that $-\alpha^{\mathrm{T}} \mathrm{Hg}(\mathbf{x}) \leq \mathrm{C}\|\mathbf{x}\|^{2-\varepsilon}$ for all $\mathbf{x}$ with $\|\mathbf{x}\| \geq \mathbf{M}$.

Our interest in systems of the form $x^{\prime}=A x+f(x)$ was stimulated by all of the work in the literature based on systems of the same form originally studied by Lorenz [ 5 ]. We hope to understand the richness of the class of chaotic systems, especially of dimension $n>3$, by classifying a sufficiently rich class of point dissipative systems in terms of their compact attractors. This paper represents a step forward in that program by enlarging the class of systems that can be first classified as point dissipative in terms of their coefficients.

\section{PRELIMINARIES}

The proof of the prinicipal result Theorem 3 uses some properties of skew symmetric matrices. These are matrices such that $A^{T}=-A$. Here are properties that are needed.

1.1. We note that $\mathbf{k}^{\mathrm{T}}$ is in the kernel of a skew symmetric $B$ relative to left multiplication if and only if $\mathbf{k}$ is in the kernel of $\mathbf{B}$ relative to right multiplication. This follows since $\left(k^{T} B\right)^{T}=-B k$ when $B$ is skew symmetric.

1.2. The rank of a skew symmetric matrix is even [ 3, page 217].

1.3. Again let $B$ be an $n$-dimensional $(n \geq 2)$ skew symmetric matrix and $C$ be the $(n-1)$ dimensional principal submatrix obtained from $B$ by removing the first row and the first column. If the $\operatorname{ker}(B)$ is nondegerate and is contained in $\left\{x \mid x_{1}=0\right\}$ then $C$ is singular. This follows since $k$ in the $k e r(B)$ implies $k_{1}=0$ that is $k=\left(0, k_{2}, \ldots, k_{n}\right)^{T}$. Now if we project $k$ by $P$ defined by $P x=\left(x_{2}, \ldots, x_{n}\right)^{T}$ then $P$ is a projection from $n$ to $n-1$ dimensional vector spaces. We note that $k$ in the kernel of $B$ implies that $P k$ is in $\operatorname{ker}(C)$. Since $k^{T}=\left(0, k_{2}, \ldots, k_{n}\right)$ we have

$$
\begin{aligned}
\mathbf{k}^{\mathrm{T}} \mathrm{B} & =\left(\mathbf{k}^{\mathrm{T}} \mathrm{B}_{1}, \mathbf{k}^{\mathrm{T}} \mathrm{B}_{2}, \ldots, \mathbf{k}^{\mathrm{T}} \mathrm{B}_{n}\right) \\
& =\left(0, \mathrm{Pk}^{\mathrm{T}} \mathrm{C}_{1}, \ldots, \mathrm{Pk}^{\mathrm{T}} \mathrm{C}_{\mathrm{n}-1}\right)=(0,0, \ldots, 0)
\end{aligned}
$$

where $B_{j}$ and $C_{j}$ refer to the $j^{\text {th }}$ column of $B$ and $C$, respectively. 
1.4. If the $\operatorname{ker}(B)$ is contained in $\left\{x \mid x_{1}=0\right\}$ then the $\operatorname{dim}(\operatorname{ker}(B))<\operatorname{dim}(\operatorname{ker}(C))$. To see this let $n$ be the dimension of $B$. We see from the proof of 3$)$ that the $\operatorname{dim}(\operatorname{ker}(C)) \geq \operatorname{dim}(\operatorname{ker}(B))$ and $\operatorname{dim}(\operatorname{ker}(C)) \geq 1$. Suppose that $\operatorname{dim}(\operatorname{ker}(C))=\operatorname{dim}(\operatorname{ker}(B))$. Then $\operatorname{rank}(C)+\operatorname{dim}(\operatorname{ker}(C))=$ $n-1$ and $\operatorname{rank}(B)+\operatorname{dim}(\operatorname{ker}(B))=n$. and so $\operatorname{rank}(C)=\operatorname{rank}(B)-1$. But this impossible since the ranks of both the skew symmetric matrices $B$ and $C$ must be even.

Without loss of generality we can assume that the hyperplane of zeros of $f$ is $\left\{x \mid x_{1}=0\right\}$. For consider the dynamical system $\quad \mathbf{x}^{\prime}=\mathbf{A x}+\mathbf{f}(\mathbf{x})$ where the zeros of $\mathbf{f}$ are an $(n-1)$ dimensional hyperplane, call it $H$. Let $R$ be a rotation so that $H$ goes onto the hyperplane $Y_{1}=$ $\left\{y \mid y_{1}=0\right\}$ under $R^{-1}$. Let $x=R y$. Then the orginal dynamical system is represented by $y^{\prime}=R^{-1} A R y+R^{-1} f(R y)$. Note that the zeros of $R^{-1} f(R y)$ are precisely the zeros of $f(x)$ under the rotation $R^{-1}$. This is true for any rotation $R$. We note that $Z\left(\mathcal{R}^{-1} f(\boldsymbol{R} y)\right) \supseteq$ $R(Z(f(x)))$. This applies to both $R$ and $R^{-1}$.

Let $x_{0} \neq 0$ be in $H$. The hypothesis of our theorem requires that $x_{0}{ }^{T} A x_{0}<0$. Then $y_{0}=$ $R x_{0}$ and $y_{0}{ }^{T}\left(R^{-1} A R\right) y_{0}=x_{0}{ }^{T} A x_{0}<0$. Since $R$ has an inverse the hypotheses for the dynamical system holds whether represented in terms of $\mathbf{x}$ or $\mathbf{y}$.

Now assuming that $Z(f)=\{x \mid x=0\}$ we notice that $f$ has a convenient representation. Ea'ch coordinate function of $f, f_{k}, k=1,2, \ldots, n$, must have $\left\{x \mid x_{1}=0\right\}$ contained in its zero set. If we represent $f_{k}, k=1,2, \ldots, n$ by

$$
f_{k}(x)=\sum_{i=j}^{n} \sum_{j=1}^{n} b_{k i j} x_{i} x_{j}
$$

then

$$
f_{k}\left(0, x_{2}, \ldots, x_{n}\right)=\sum_{i=j}^{n} \sum_{j=1}^{n} b_{k i j} x_{i} x_{j}=0 \quad \text { for all } x_{2}, x_{3}, \ldots, x_{n}
$$

Therefore, $b_{k i j}=0$ if neither $i$ nor $j$ is 1 . Or

$$
f_{k}(x)=\sum_{j=1}^{n} b_{k 1 j} x_{1} x_{j}=x_{1} \sum_{j=1}^{n} b_{k 1 j} x_{j}
$$

and

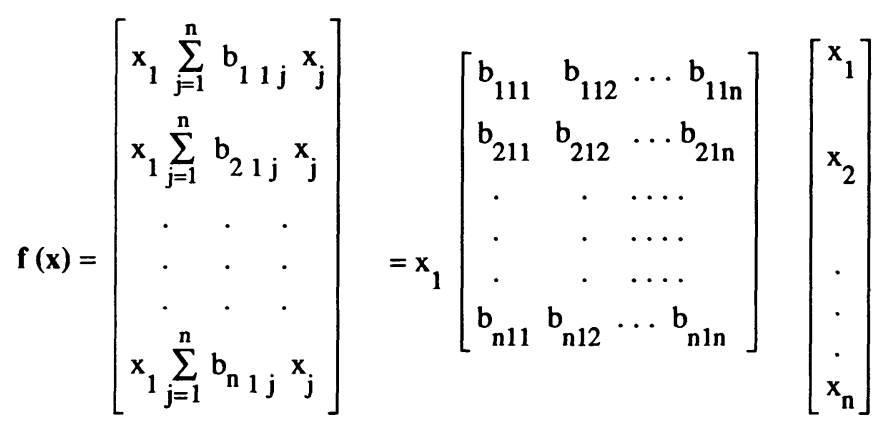

Let $B$ denote the matrix $\left(b_{k 1}\right)_{11}^{n n}$. Since $x^{T} f(x)=x_{1} \quad x^{T} B x=0$ for all $x$ 
$\mathbf{x}^{\mathrm{T}} \mathbf{f}(\mathbf{x})=\sum_{\mathrm{j}=1}^{\mathrm{n}} \mathrm{b}_{\mathrm{j} 1 \mathrm{j}} \mathbf{x}_{1} \mathbf{x}_{j}^{2}+\sum_{\mathbf{k}=1}^{\mathrm{n}} \sum_{\mathrm{j}=1}^{\mathrm{n}}\left(\mathrm{b}_{\mathbf{k} 1 \mathrm{j}}+\mathrm{b}_{\mathrm{j} 1 \mathrm{k}}\right) \mathrm{x}_{1} \mathbf{x}_{\mathrm{j}} \mathbf{x}_{\mathbf{k}}=0$ which is the zero polynomial having zero coefficients. B is skew symmetric and we drop the second subscript which is always one and we have the representation

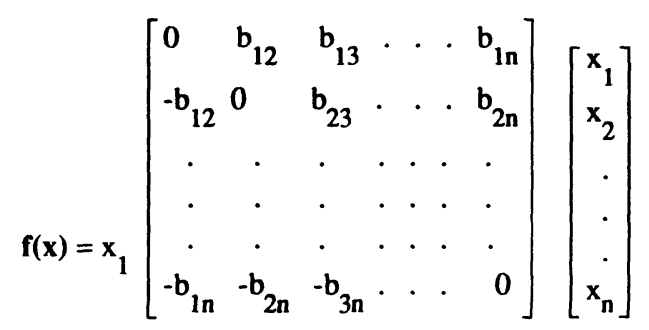

We use the Lyapunov function $V(x)=\frac{1}{2}(x-\alpha)^{T}(x-\alpha)$ then $\frac{d(V(x)(T)))}{d t}=x^{T} A x-\alpha^{T} f(x)+$ linear terms. For large $\|x\|$ the quadratic terms $x^{T} A x-\alpha^{T} f(x)$ dominate $\frac{d(V(x(t))}{d t}$ therefore our quest is find an $\alpha$ which turns the quadratic into a negative definite function.

\section{EXTENSION OF PREVIOUS RESULTS}

A sufficient condition for a quadratic dynamical system to be point dissipative has been given [ 1 ] when the dimension is two or three. This condition uses a relation between the quadratic and linear parts of the system when $f$ is conservative. The following lemma allows us to extend the condition to the case where there exists a positive definite matrix $S$ such that Sf is conservative.

Lemma 1. Let

$$
\mathbf{x}^{\prime}=\mathrm{Ax}+\mathbf{f}(\mathbf{x})
$$

be a quadratic dynamical system for which there exists a positive definite matrix $S$ such that $S f$ is conservative. Then there exists matrix $\mathrm{H}$ for which the change of variables $\mathbf{y}=\mathbf{H x}$ transforms the dynamical system (2.1) into $\mathbf{y}^{\prime}=\mathrm{By}+\mathbf{g}(\mathbf{y})$ which has a conservative quadratic term. Furthermore, $\mathbf{x}^{\mathrm{T}} \mathrm{SAx}=\mathbf{y}^{\mathrm{T}} \mathbf{B y}$.

Proof. We can factor $S$ by $S=H^{T} H$ [3]. Let $y=H x$ or $x=H^{-1} y$. The system transforms into $\mathbf{y}^{\prime}=\left(\mathrm{HAH}^{-1}\right) \mathbf{y}+\mathbf{H} \mathbf{f}\left(\mathrm{H}^{-1} \mathbf{y}\right)$ or

$$
\mathbf{y}^{\prime}=\mathbf{B y}+\mathbf{g}(\mathbf{y})
$$

The system (2.2) has a conservative quadratic term since $\mathbf{y}^{\mathrm{T}} \mathbf{g}(\mathbf{y})=\mathbf{x}^{\mathrm{T}} \mathbf{H}^{\mathrm{T}} \mathbf{H} \mathbf{f}(\mathbf{x})=\mathbf{x}^{\mathrm{T}} \mathbf{S f}(\mathbf{x})=0$. Note that $\mathbf{x}^{\mathrm{T}} \mathrm{SAx}=\mathbf{y}^{\mathrm{T}}\left(\mathrm{H}^{-1}\right)^{\mathrm{T}} \mathbf{H}^{\mathrm{T}} \mathrm{HA} \mathrm{H^{-1 }} \mathbf{y}=\mathbf{y}^{\mathrm{T}} \mathrm{HA} \mathrm{H}^{-1} \mathbf{y}=\mathbf{y}^{\mathrm{T}} \mathrm{By}$.

When $\mathrm{Sf}$ is conservative then we say that a vector $\alpha$ is admissible for the dynamical system $x^{\prime}=A x+f(x)$ if $-x^{T} S A x+\alpha^{T} H f(x)$ is a positive definite function where $S=H^{T} H$. 
When $f$ is conservative then the condition for an admissible $\alpha$ reduces to $-x^{T} A x+\alpha^{T} f(x)$ is positive definite. The proofs of the theorems when the quadratic part of the system is conservative entail demonstrating the existence of an admissible $\alpha$ for the system.

These results [ 1 ] can be restated as follows:

Theorem 1. A quadratic dynamical system $x^{\prime}=A x+f(x)$ is point dissipative when there exists a positive definite matrix $S$ such that $S f$ is conservative and there exists an admissible $\alpha$. If the system has dimension 2 or $3, \mathrm{Sf}$ is conservative and $\mathrm{z}^{\mathrm{T}} \mathrm{SAz}<0$ for any $\mathrm{z}$ which is a nontrival zero of $f$ then the system is point dissipative.

Proof. We can transform the dynamical system by $y=H x$ where $S=H^{T} H$ and by Lemma 1 the resulting dynamical system satisfies the hypothesis of the previous theorem. Hence, the resulting dynamical system as well as the original system are both point dissipative.

Another direction of generalizing the past results is to consider nonlinear dynamical systems which have nonquadratic nonlinear terms as well as quadratic terms. Relative to the nonlinear terms there again must exits a positive definite matrix $S$ such that the nonlinear terms premultiplied by $S$ are conservative.

Theorem 2. When there exists a positive definite matrix $S$ such that $\mathbf{S g}$ and the quadratic function $\mathrm{Sf}$ are conservative and

Condition (A) For some admissible $\alpha$ for $x^{\prime}=A x+f(x)$ there exists an ordered triple of numbers $(\varepsilon, C, M)$ such that $-\alpha^{T} H g(x) \leq C\|x\|^{2-\varepsilon}$ for all $x$ with $\|x\| \geq M$.

then

is point dissipative.

$$
\mathbf{x}^{\prime}=A \mathbf{x}+\mathbf{f}(\mathbf{x})+\mathbf{g}(\mathbf{x})
$$

Note that condition (A) can be replaced by either of the stronger conditions (B) or (C).

Condition (B) There is an admissible $\alpha$ for $x^{\prime}=A x+f(x)$ and $\|\mathbf{g}\|=0\|x\| 2$.

Condition (C) There is an admissible $\alpha$ for $\mathbf{x}^{\prime}=A \mathbf{x}+\mathbf{f}(\mathbf{x})$ and $\mathbf{g}$ is bounded.

\section{THE PROOF OF THE THEOREM}

Theorem 3. A quadratic dynamical system $\mathbf{x}^{\prime}=\mathbf{A x}+\mathbf{f}(\mathbf{x}), \mathrm{A}$ a matrix and $\mathbf{f}$ a quadratic function, is point dissipative when

(1) there exists a positive definite matrix $S$ such that $S f$ is conservative,

(2) the zeros of $f$ are an $(n-1)$-dimensional hyperplane, and

(3) $\mathbf{z}^{\mathrm{TSAz}}<0$ for any $\mathbf{z}$ which is a nontrivial zero of $\mathbf{f}$.

Proof. From Lemma 1 the system can be transformed into a system which has a conservative quadratric term. Moreover, the zero properties are preservered. So we assume that $f$ is conservative. We can assume that $Z(f)=\left\{x \mid x_{1}=0\right\}$ and $f(x)=x_{1} B x$ where $B$ is 
skew symmetric as shown in Section 1.3. Since $Z(f)=\left\{x \mid x_{1}=0\right\}, k$ in the kernel of $B$ implies $\left(0, k_{2}, \ldots, k_{n}\right)$ or that $k_{1}=0$. Let $h$ be a vector which is not in the kernel of $B$ but $\left(h_{2}, \ldots, h_{n}\right)^{T}$ is in the $\operatorname{ker}(C)$, see Section 1.4. Here again $C$ is the principal submatrix of $B$ formed by removing the first row an column of $B$. Moreover, if the rank of $C \neq 0$, we can choose $h$ so that $h_{1}=0$.

Let $G=\{\alpha \mid \alpha=t h, t$ a real number $\}$ and we will show $G$ is contained in the set of admissible $\alpha$ 's. Note that if $\alpha$ is in $G$ then $\alpha^{\mathrm{T}} \mathrm{f}(\mathrm{x})=\mathrm{x}_{1}$ th $^{\mathrm{T}} \mathrm{B} x=\mathrm{x}_{1} \mathrm{t}(\mathrm{q}, 0,0, \ldots, 0) \mathrm{x}=\mathrm{tq}_{1}^{2}$ since $P h$ is in the $\operatorname{ker}(C)$. Also $q \neq 0$ since $h$ is not in the $\operatorname{ker}(B)$. Notice that $q \neq 0$ whether or not the $\operatorname{rank}(C)=0$ furthermore, we can choose $t$ so that $\alpha^{T} f(x) \geq 0$ for all $x$.

We can restrict our attention to the sphere $\|x\|=1$ which is compact. There is a closed cone on a closed cone which contains the hyperplane $\left\{x \mid x_{1}=0\right\}$ such that $x^{T} A x<0$ for all $x \neq$ 0 in this cone. Hence, there is an $\varepsilon>0$ such that $x^{T} A x<0$ on $\left\{x \mid\|x\|=1\right.$ and $\left.-\varepsilon \leq x_{1} \leq \varepsilon\right\}$. Let $M=\max \left(x^{T} A x\right)$ on $\{x \mid\|x\|=1\}$. By picking $t$ large enough in magnitude we can assure that tq $\varepsilon^{2}>M$. Hence, $\alpha^{\mathrm{T}} \mathbf{f}(\mathbf{x}) \geq \operatorname{tq} \varepsilon^{2}>\mathbf{M}$ on $\left\{\mathbf{x} \mid\|\mathbf{x}\|=1\right.$ and $\left.\left|x_{1}\right| \geq \varepsilon\right\}$. Thus for all of $\{\mathbf{x} \mid$ $\|x\|=1\}, x^{T} A x-\alpha^{T} f(x)<0$. And so for all nonzero $x$ we have that $x^{T} A x-\alpha^{T} f(x)<0$ which implies that the system is point dissipative.

This result can be generalized by adding to the differential equation any conservative function $\mathbf{g}(\mathbf{x})$ whose growth is restricted. The corollary states this condition.

Corollary Let $g$ and the quadratic function be conservative. If $Z(f)$ is an (n-1)-hyperplane and $\mathbf{x}$ in $\mathbf{Z ( f )}$ implies $\mathbf{x}^{\mathrm{T}} \mathrm{A} \mathbf{x}<0$ and

Condition (A) for some admissible $\alpha$ there exists an ordered triple of numbers $(\varepsilon, C, M)$ such that $-\alpha^{\mathrm{T}} \mathbf{g}(\mathbf{x}) \leq\|x\|^{2-\varepsilon}$ for all $\mathbf{x}$ with $\|\mathbf{x}\| \geq \mathbf{M}$.

then

is point dissipative.

$$
x^{\prime}=A x+f(x)+g(x)
$$

Note that condition (A) can be replaced by either of the stronger conditions (B) or (C).

Condition (B) There is an admissible $\alpha$ for $x^{\prime}=A x+f(x)$ and $\|g\|=0\|x\| 2$.

Condition (C) There is an admissible $\alpha$ for $\mathbf{x}^{\prime}=A \mathbf{x}+\mathbf{f}(\mathbf{x})$ and $g$ is bounded.

\section{EXAMPLES}

Consider the dynamical system

$$
\mathbf{x}^{\prime}=\left[\begin{array}{rrrr}
2 & -1 & -1 & -1 \\
-1 & -3 & -1 & -1 \\
-1 & -1 & -3 & -1 \\
-1 & -1 & -1 & -3
\end{array}\right] \mathbf{x}+\left[\begin{array}{l}
x_{1} x_{2}+x_{1} x_{3}+x_{1} x_{4} \\
-x_{1}^{2}+x_{1} x_{3}+x_{1} x_{4} \\
-x_{1}^{2}-x_{1} x_{2}+x_{1} x_{4} \\
-x_{1}^{2}-x_{1} x_{2}-x_{1} x_{3}
\end{array}\right]
$$

In this example the conservative quadratic function can be writen as 


$$
\mathbf{f}(\mathbf{x})=x_{1}\left[\begin{array}{cccc}
0 & 1 & 1 & 1 \\
-1 & 0 & 1 & 1 \\
-1 & -1 & 0 & 1 \\
-1 & -1 & -1 & 0
\end{array}\right] \mathbf{x}=x_{1}
$$

$B$ is a nonsingular matrix and $C=B_{11}$ is singular. The kernel of $C$ is generated by $(1,-1,1)$. Since $B$ is nonsingular the zero set of $f(x)$ is $Z(f)=\left\{z \mid z=\left(0, z_{2}, z_{3}, z_{4}\right)=\left\{x \mid x_{1}=0\right\}\right.$. The hypothesis of the theorem hold since

$$
\left(0, z_{2}, z_{3}, z_{4}\right) A\left[\begin{array}{l}
0 \\
z_{2} \\
z_{3} \\
z_{4}
\end{array}\right]=\left(z_{2}, z_{3}, z_{4}\right) A_{11}\left[\begin{array}{l}
z_{2} \\
z_{3} \\
z_{4}
\end{array}\right] \text { and } A_{11}=\left[\begin{array}{ccc}
-3 & -1 & -1 \\
-1 & -3 & -1 \\
-1 & -1 & -3
\end{array}\right]
$$

is a negative definite matrix. We can use $\alpha^{\mathrm{T}}=(0, t,-t, t)$ and $\alpha^{\mathrm{T}} \mathbf{f}(\mathbf{x})=\mathrm{x}_{1}(0, t,-t, t) \cdot\left(\mathrm{x}_{2}+\mathrm{x}_{3}+\right.$ $\left.\mathrm{x}_{4},-\mathrm{x}_{1}+\mathrm{x}_{3}+\mathrm{x}_{4}, \mathrm{x}_{1}+\mathrm{x}_{2}-\mathrm{x}_{4},-\mathrm{x}_{1}-\mathrm{x}_{2}-\mathrm{x}_{3}\right)^{\mathrm{T}}=\mathrm{tx}_{1}{ }^{2}$. So the quadratic term of the Lyapunov function is

$$
x^{T} A x-\alpha^{T} f(x)=x^{T} A x-t_{1}^{2} x^{T}(A-Q) x=x^{T}\left[\begin{array}{cccc}
2-t & -1 & -1 & -1 \\
-1 & -3 & -1 & -1 \\
-1 & -1 & -3 & -1 \\
-1 & -1 & -1 & -3
\end{array}\right] x
$$

This quadratic function is negative definite when $t>2.6$. By the theorem this nonlinear quadratic dynamical system is point dissipative.

Indeed in the example $q$ turns out to be 1 . Therefore, $\alpha^{T} f(x)$ can only change the $a_{11}$ element of A. This turns out be just what we need and want. Because the zero set of $f$ is $\{(0$, $\left.z_{2}, z_{3}, z_{4}\right)$ \} we must have that $-A_{11}$ is positive definite. If $t$ can be choosen so that $\operatorname{det}(-A+$ Q) is positive then that will be enough to insure that $-A+Q$ is positive definite.

Let us choose $t=3$ and return to the derivative of the Lyapunov function. We can set it equal to zero and have the equation of a ellipsoid which contains the attractor of the system. The equation is

$$
\mathbf{x}^{\mathrm{T}}\left[\begin{array}{rrrr}
-1 & -1 & -1 & -1 \\
-1 & -3 & -1 & -1 \\
-1 & -1 & -3 & -1 \\
-1 & -1 & -1 & -3
\end{array}\right] \mathbf{x}-(0,3,-3,3)\left[\begin{array}{cccc}
2 & -1 & -1 & -1 \\
-1 & -3 & -1 & -1 \\
-1 & -1 & -3 & -1 \\
-1 & -1- & -1 & -3
\end{array}\right] \mathbf{x}=0
$$

If we use the rotation

$$
\mathbf{x}=\mathbf{R y}=\left[\begin{array}{llll}
0.0000 & 0.0000 & 0.9370 & 0.3493 \\
0.8165 & 0.0000 & -0.2017 & 0.5410 \\
-0.4082 & 0.7071 & -0.2017 & 0.5410 \\
-0.4082 & 0.7071 & -0.2017 & 0.5410
\end{array}\right] \mathbf{x}
$$

the above equation becomes 


$$
\mathbf{y}^{\mathrm{T}}\left[\begin{array}{rrcr}
-2 & 0 & 0 & 0 \\
0 & -2 & 0 & 0 \\
0 & 0 & -3+\sqrt{7} & 0 \\
0 & 0 & 0 & -3-\sqrt{7}
\end{array}\right] \mathbf{y}+(0.3330,7.9233,10.61307,-2.1149) \mathbf{y}=0
$$

or

$$
2\left(x_{1}-0.16651\right)^{2}+2\left(x_{2}-3.9617\right)^{2}+0.3542\left(x_{3}-5.3065\right)^{2}+5.6458\left(x_{4}-1.0574\right)^{2}=47.7333
$$

The distance to the center is 6.7082 and the half-diameter of the ellipsiod is 11.6070 and our bound is the sum of these two numbers is $\mathbf{1 8 . 3 1 6 2}$

Consider the dynamical system

$$
x^{\prime}=\left[\begin{array}{ll}
-4 & 4 \\
-4 & 1
\end{array}\right] x+\left[\begin{array}{c}
6 x y-4 y^{2} \\
-6 x^{2}+4 x y
\end{array}\right]+\left[\begin{array}{c}
2 y e^{-x-y} \\
-2 x e^{-x-y}
\end{array}\right]
$$

where the linear part Ax and the quadratic function

$$
f(x)=\left[\begin{array}{c}
6 x y-4 y^{2} \\
-6 x^{2}+4 x y
\end{array}\right]
$$

are related by $\mathbf{z}^{\mathrm{T}} \mathbf{A z}<0$ when $\mathbf{z}$ is a nontrival zero of $\mathbf{f}$. The admissible $\alpha$ 's are those for which $\mathbf{x}^{\mathrm{T}} \mathrm{Ax}-\alpha^{\mathrm{T}} \mathbf{f}(\mathbf{x})$ is a negative definite function. This is equivalent to the matrix

$$
\left[\begin{array}{lr}
4-6 \alpha_{2} & 3 \alpha_{1}+2 \alpha_{2} \\
3 \alpha_{1}+2 \alpha_{2} & -1-4 \alpha_{1}
\end{array}\right]
$$

being poistive definite. For the diagonial terms to be positive definite $\alpha_{1}<-1 / 4, \alpha_{2}<2 / 3$ and for the determiniant of the matrix to be positive

$$
-9 \alpha_{1}^{2}+12 \alpha_{1} \alpha_{2}-4 \alpha_{2}^{2}-16 \alpha_{1}+6 \alpha_{2}-4>0
$$




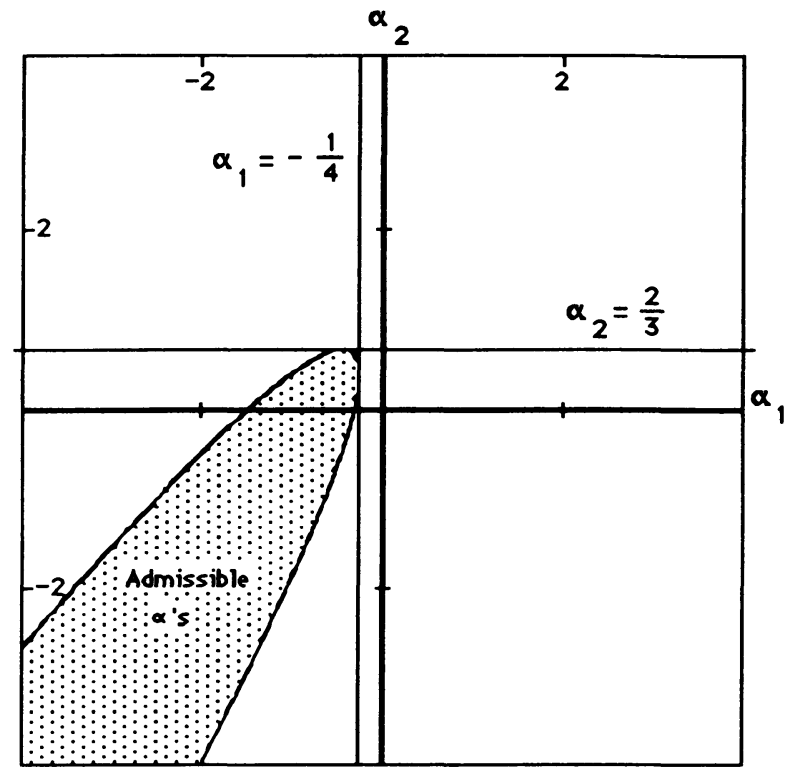

Figure 1

The shaded region is the set of admissible $\alpha^{\mathrm{T}}$ relative to $\mathrm{A}$ and $\mathrm{f}$. Now if for one of these $\alpha$ 's condition (A) hold the the dynamical system is point dissipative. This is the case when we choose $\alpha^{\mathrm{T}}=(-1 / 2,1 / 2)$

$$
-\alpha^{T} g(x)=-\left(\frac{-1}{2}, \frac{1}{2}\right)\left[\begin{array}{c}
2 x e^{-x-y} \\
-2 y e^{-x-y}
\end{array}\right]=-(x+y) e^{-x-y} \leq \frac{1}{e}
$$

and the value of the determinate is 0.75 and the diagonal elements are 1 and 1 . Here is the graph [6] of some of the trajectories.

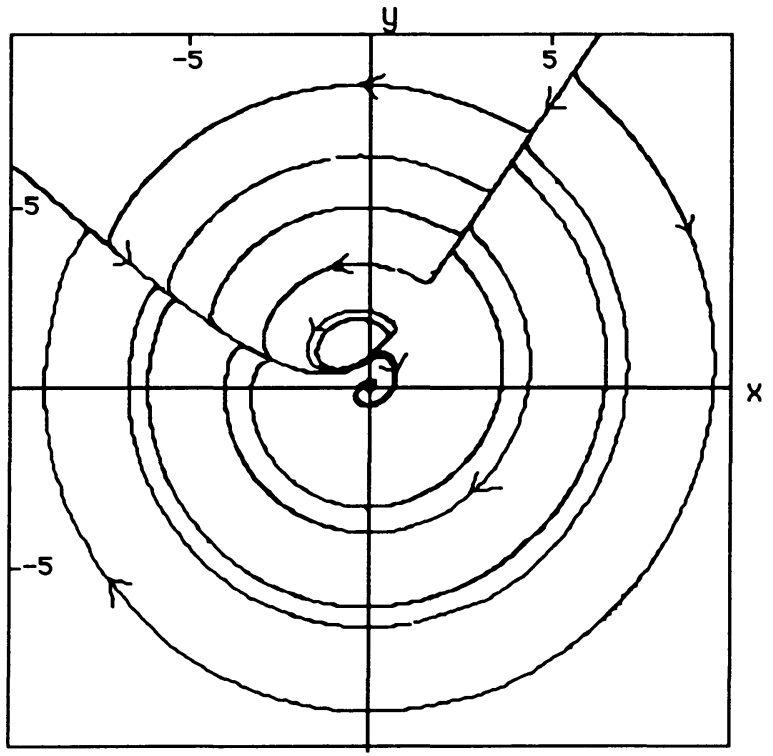

$$
\begin{aligned}
& \frac{d x}{d t}=-4 x+4 y+6 x^{2}-4 x y+2 x e^{-x-y} \\
& \frac{d y}{d t}=-4 x+y-6 x y+4 y^{2}-2 y e^{-x-y}
\end{aligned}
$$

Figure 2 
The attractor above the origin is magnified in the following Figure 3. It indicates that there is an attractor at $(-0.5,1)$ surrounded by a limit cycle which is an repeller. There is also an attractor at $(0,0)$ and a saddle point near $(1,2)$.

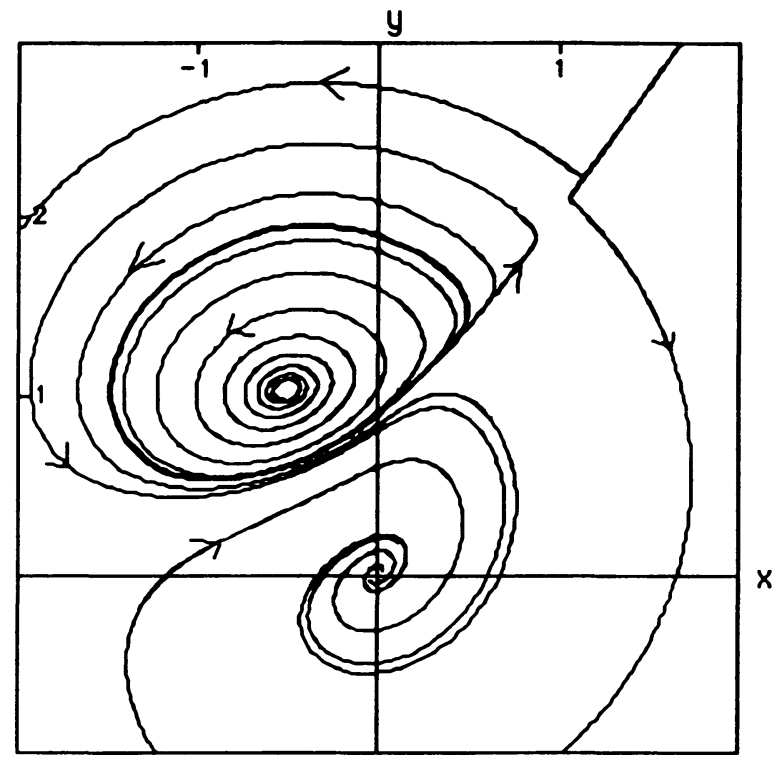

Figure 3

\section{BIBLIOGRAPHY}

1. BOSE, A. K., A. S. Cover and J. A. Reneke, On Point-Dissipative Systems of Differential Equations with Quadratic Nonlinearity, Int. J. Math. and Math. Sci., 14(1990), 99-110.

2. HALE, J. K., L. T. Magalhaes and W. M. Oliva, An Introduction to Infinite Dimensional Dynamical Systems, Geometric Theory, New York, Berlin, Heidelberg, Tokyo, Applied Mathematical Sciences, Springer-Verlag.

3. HORN, R. A. and C. R. Johnson, Matrix Analysis, Cambridge, Cambridge University Press, 1985.

4. LASALLE, Joseph and Solomon Lefschetz, Stability by Liapunov's Direct Method, with Applications, New York, Academic Press, 1961.

5. LORENZ, E. N., Deterministic non-periodic flow, J. Atmos. Sci., 20(1963), 130-141.

6. Phase Portraits, Version 2.0, Department of Mathematics and Computer Science, Drexel University, 1988. 


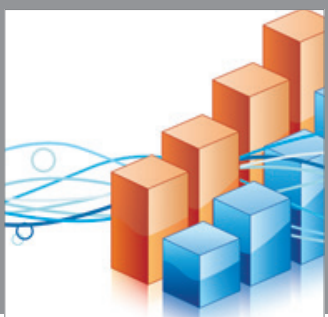

Advances in

Operations Research

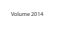

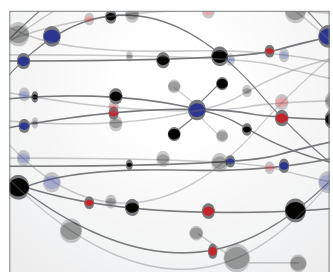

\section{The Scientific} World Journal
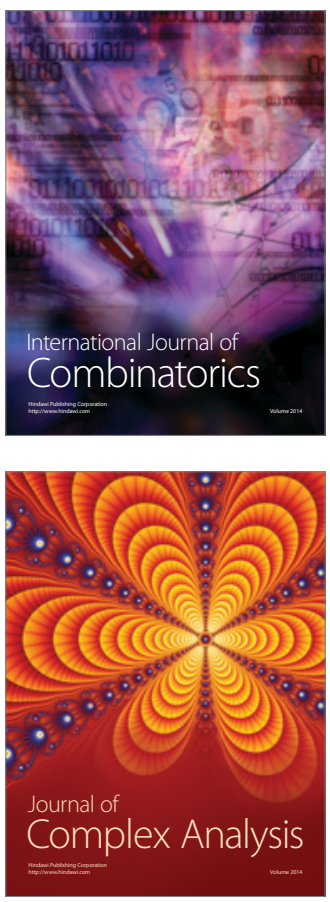

International Journal of

Mathematics and

Mathematical

Sciences
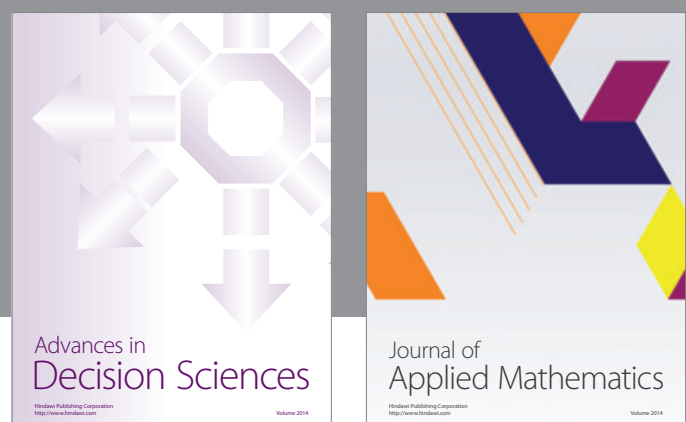

Journal of

Applied Mathematics
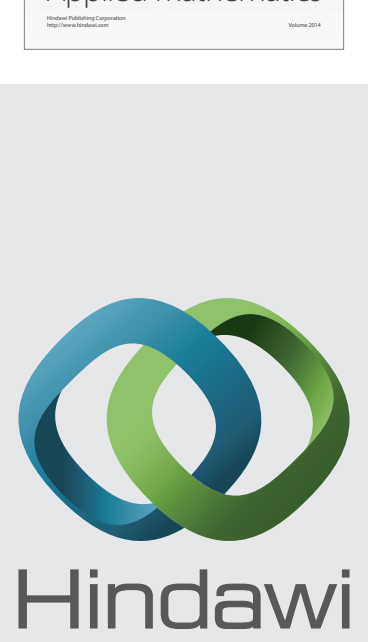

Submit your manuscripts at http://www.hindawi.com
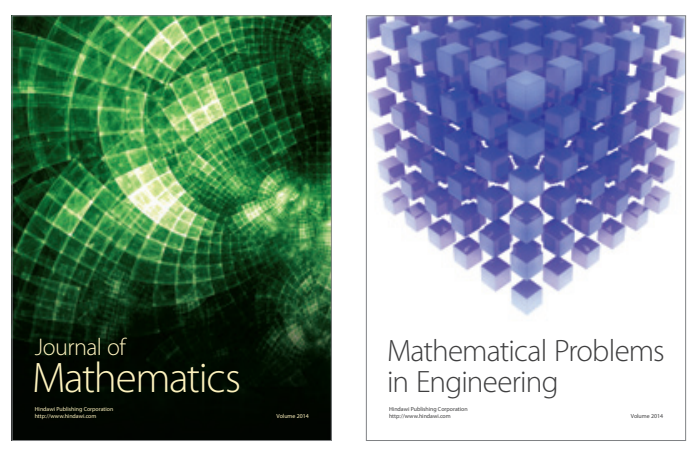

Mathematical Problems in Engineering
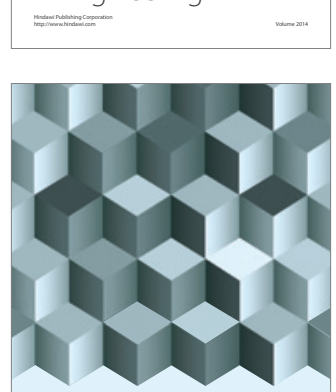

Journal of

Function Spaces
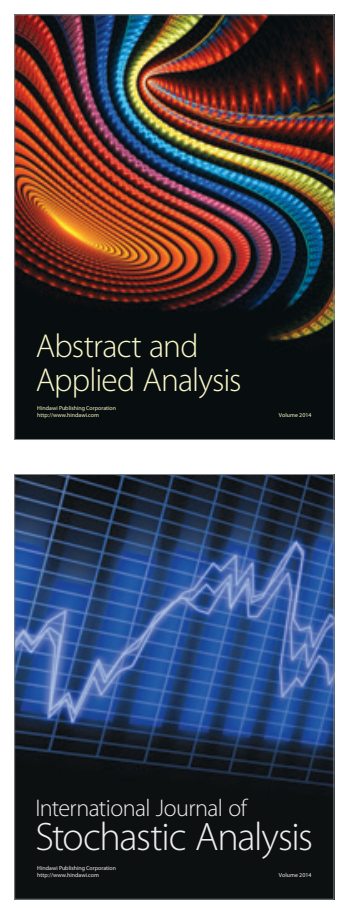

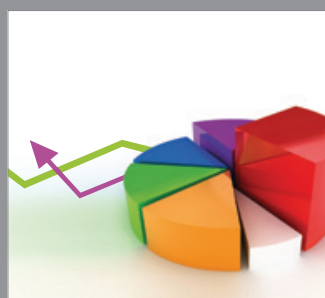

ournal of

Probability and Statistics

Promensencen
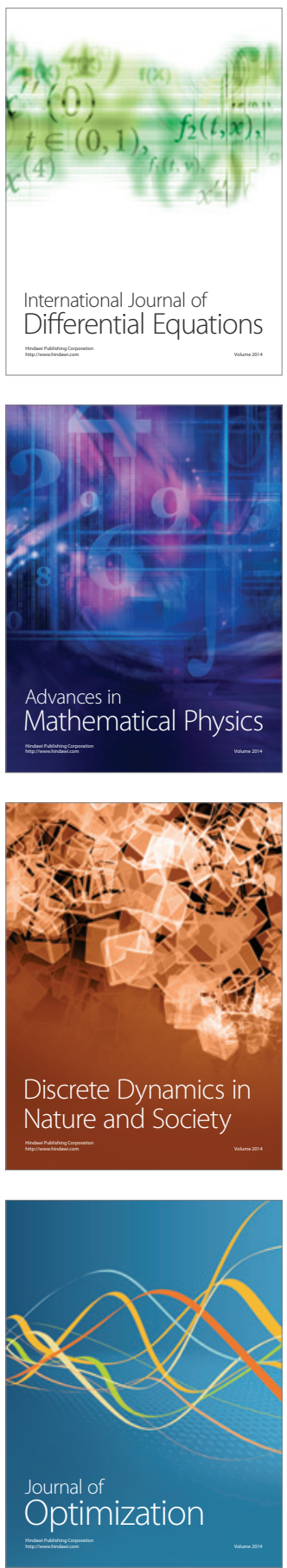